\title{
Avaliação nutricional em pacientes infectados pelo Vírus da Imunodeficiência Adquirida
}

\section{Nutritional assessment in patients infected with Human Immunodeficiency Virus}

Rosana Mendes Reis BARBOSA ${ }^{1}$

Nélida Schmid FORNÉS²

\section{RE S U M O}

Pacientes infectados com o Vírus da Imunodeficiência Humana ou com Síndrome da Imunodeficiência Adquirida freqüentemente apresentam desnutrição protéico-energética, com manifestações heterogêneas, as quais dependem do estado de saúde prévio do paciente. O estado nutricional deve ser avaliado e monitorado cuidadosamente para que o plano terapêutico possa ser efetivo. Uma avaliação nutricional abrangente é fundamental; além disso, toda a equipe de profissionais da saúde e os próprios pacientes devem estar atentos para uma ação precoce e integrada.

Termos de indexação: nutrição, Síndrome de Imunodeficiência Adquirida, avaliação nutricional, desnutrição protéico-energética.

\section{A B S T R A C T}

Patients infected with the Human Immunodeficiency Virus or with the Acquired Immunodeficiency Syndrome often present protein-energy malnutrition. The manifestations are heterogeneous and depend on the patient's previous health status. The nutritional status must be assessed and carefully controlled, so that satisfactory results can be achieved and the therapeutic plan can be effective. A complete detailed nutritional assessment is fundamental; besides, the health professional team and the patients themselves need to be aware of the symptoms and the disease progress, in order to take an early and integrated action.

Index terms: nutrition, Acquired Immunodeficiency Syndrome, nutritional assessment, protein-energy malnutrition.

\footnotetext{
1 Nutricionista, Hospital Dr. Anuar Auad Hospital de Doenças Tropicais de Goiânia.

2 Faculdade de Nutrição, Universidade Federal de Goiás. Rua 227, Q. 68, s/n, Setor Leste Universitário, 74605-080, Goiânia, GO, Brasil. Correspondência para/Correspondence to: N.S. FORNÉS. E.-mail: nelida@fanut.ufg.br
} 


\section{N T R O D U Ç Ã O}

Diante da crescente incidência da Síndrome da Imunodeficiência Adquirida (AIDS) no Brasil, com cerca de 200 mil casos notificados ${ }^{1}$ e de acordo com as constatações de Kotler (1992)2, torna-se importante conhecer o impacto da doença no estado nutricional dos pacientes infectados pelo Human Immunodeficiency Virus (HIV), promovendo a melhora do estado nutricional, da saúde e da qualidade de vida, diminuindo o número, o tempo e o custo das hospitalizações e prolongando a sobrevivência.

A AIDS é uma doença crônica e progressiva, com impacto no estado nutricional manifestado mais agressivamente na Síndrome Consumptiva, sendo este um critério definidor de AIDS. Este impacto é notado de forma mais branda na vasta maioria dos pacientes, devendo ser prevenido ou tratado, pois mesmo a desnutrição moderada tem um claro efeito adverso no resultado da doença ${ }^{3}$.

Apesar dos importantes avanços no tratamento com a terapia antiretroviral, na quantificação da carga viral e na profilaxia de doenças agudas, Shevitz \& Knox $(2001)^{4}$ referem que pessoas com HIV comumente experimentam problemas nutricionais como perda de peso, redistribuição de gordura e obesidade. A incidência da desnutrição tende a aumentar devido à sobrevivência prolongada, sendo essencial monitorar anualmente o peso dos pacientes assintomáticos e freqüentemente daqueles sintomáticos ou com mudança de peso.

A ampla e complexa resposta metabólica ao HIV, segundo Singer et al. (1992) $)^{5}$ e Gramlich \& Mascioli (1995) ${ }^{6}$, inclui febre, hipermetabolismo, leucopenia, uso inapropriado dos substratos metabólicos com ciclos fúteis, proteólise muscular, síntese hepática de proteínas reacionais de fase aguda, gerando aumentada gliconeogênese e lipogênese e diminuindo a síntese de albumina. A produção de citoquinas e as alterações dos hormônios tiroidianos podem estar associadas à exacerbação do consumo corporal, anorexia e caquexia. Por outro lado, a redução da atividade física causada pela astenia não é suficiente para conservar energia e evitar a perda de peso.

A Avaliação Nutricional (AN) evidencia deficiências isoladas ou globais de nutrientes e possibilita a classificação dos indivíduos em níveis graduados de estado nutricional, servindo como um valioso instrumento para a determinação da terapêutica clínica ou dietética, a fim de tentar corrigir o déficit observado ${ }^{4,7}$.

A atenção aos problemas de desnutrição é de primordial importância devido ao tempo de morte ser mais exatamente relativo ao grau de depleção de Massa Celular Corporal (MCC) do que a alguma infecção basal específica². O tipo de orientação nutricional, a complexidade da avaliação e o grau de intervenção devem variar com o estágio da doença do indivíduo.

Este artigo apresenta a aplicação prática dos métodos de avaliação do estado nutricional em pacientes infectados pelo HIV/AIDS, podendo ser utilizado para subsidiar a assistência prestada a eles.

\section{PROPÓSITO DA AVALIAÇÃ O DO ESTADO NUTRICIONAL E M HIVIAIDS}

A avaliação do estado nutricional, de acordo com a posição da American Dietetic Association $^{7}$, é fundamental para o adequado diagnóstico da Desnutrição Energético-Protéica (DEP) ou para a identificação de fatores de risco e para a instituição efetiva da Terapia Nutricional (TN), a fim de melhorar o estado nutricional, a sobrevida e a qualidade de vida dos pacientes com AIDS, freqüentemente acometidos por desordens nutricionais e consumptivas, as quais ocorrem em "cascata" como parte da resposta imune à infecção, resultando em preferencial e rápido consumo da Massa Corporal Magra (MCM).

A AN nos estágios precoces da doença é particularmente útil para indivíduos assintomáticos, 
nos quais o sistema imunológico ainda não está irreversivelmente comprometido, podendo ser comparada aos valores basais dos padrões atualmente aceitos para indivíduos saudáveis

O estado nutricional orienta-se por fatores estáticos e dinâmicos, necessitando de medidas individualizadas e longitudinais, levando-se em consideração vários indicadores. Estudos de McCorkindale et al. (1990) e Babameto \& Kotler $(1997)^{10}$, sobre composição corporal em pacientes nas fases mais precoces da AIDS, demonstram depleção significativa da MCC e reduções no peso e no conteúdo de gordura corporal. O padrão de depleção tecidual se parece mais com um estado de estresse ou injúria do que com simples fome; porém, pode haver um subgrupo no qual sobressai o padrão de fome, geralmente associado com disfunção gastrointestinal primária.

\section{Estágios da doença}

Referindo-se aos estágios da AIDS, Melchior et al. (1993) ${ }^{11}$, Ott et al. (1993) ${ }^{12} \mathrm{e}$ Davidhizar \& Dunn (1998) ${ }^{13}$ descrevem que no estágio precoce há diminuição da MCC e aumento da água extracelular (AE), sem perda de peso. No estágio intermediário, a MCC diminui mais, a $A E$ aumenta e ocorre perda de peso. No estágio tardio, as taxas de perda de MCC e de $A E$ aumentam mais, a gordura corporal diminui e ocorre severa perda de peso, características que se agravam na presença de infecções agudas. Análises regressivas de perda de peso corporal,
MCC e albumina, independentemente da contagem de células CD4, são preditoras de morte na AIDS, progressão da doença com maior predisposição a doenças agudas e hospitalizações freqüentes.

Todo paciente deve ser avaliado completamente para determinar seu estado nutricional considerando o estágio da doença (Quadro 1) ${ }^{14}$.

De acordo com Singer et al. $(1992)^{5}$, a simples medição do peso corporal minimiza a perda de $\mathrm{MCM}$, devido à relativa expansão extracelular de água, e o estágio da doença deve ser considerado, pois é um fator chave na eficácia da TN.

\section{Padrões de perda de peso}

Segundo Carbonnel \& Cosnes (1997) ${ }^{15}$, pacientes com AIDS podem emagrecer rapidamente ( $5 \mathrm{~kg}$ por mês, em média), indicando uma associação a uma infecção secundária sistêmica em mais de $80 \%$ dos casos, ou emagrecer mais lentamente $(1 \mathrm{~kg}$ por mês, em média), indicando uma doença gastrintestinal (candidíase, criptosporidiose, ou outra) ou redução na ingestão alimentar. Na prática, situações diversas são defrontadas, sendo mais freqüentes: um emagrecimento progressivo associado à diarréia crônica; ausência de recuperação de peso, apesar de tratamento medicamentoso eficaz e TN; emagrecimento progressivo sem causa conhecida;

Quadro 1. Aspectos a serem observados de acordo com o estágio da doença.

\begin{tabular}{|c|c|c|}
\hline Estágio & CD4 & Características \\
\hline Precoce & Acima de 500 células $/ \mathrm{mm}^{3}$ & $\begin{array}{l}\text { Aumento no gasto energético e mudanças no estoque corporal de vitamina } \\
\mathrm{B}_{12} \text { e folato. }\end{array}$ \\
\hline Intermediário & Entre 200 e 500 células $/ \mathrm{mm}^{3}$ & $\begin{array}{l}\text { Associação com deficiências nutricionais específicas de vitamina } B_{12} \text {, folato, } \\
\text { zinco e selênio. Provavelmente ocorre um ciclo de ingestão alimentar flutuante, } \\
\text { nutrição sub-ótima e maior suscetibilidade a infecções. }\end{array}$ \\
\hline Tardio & Abaixo de 200 células $/ \mathrm{mm}^{3}$ & $\begin{array}{l}\text { Relação aumentada com risco para perda de peso severa e intratável, resultado } \\
\text { em desnutrição e fadiga crônica. A ocorrência de enteropatias agudas e/ou } \\
\text { crônicas e infeccões aqudas, bem como Síndrome Consumptiva, é maior. }\end{array}$ \\
\hline
\end{tabular}


emagrecimento maciço nos doentes em fase terminal.

A perda de peso crônica está associada ao hipermetabolismo e à má absorção que, em geral, aparece em indivíduos sem infecções agudas, e seu tratamento é mais difícil. A perda de peso aguda está relacionada quase exclusivamente com a presença de infecções. Sua causa fundamental é a diminuição da ingestão e pode preceder em quinze a trinta dias aos sintomas próprios da infecção, constituindo um sinal de alerta ${ }^{11,16}$. Durante os episódios de perda de peso, o gasto energético total fica reduzido, porém o principal determinante é a baixa ingestão alimentar, observada na síndrome consumptiva associada à AIDS ${ }^{17}$.

\section{Anamnese alimentar}

A classificação do risco de desnutrição é uma importante ferramenta para estabelecer o perfil clínico do paciente e a intervenção nutricional adequada para prevenir o consumo da MCM. O nutricionista deve conduzir a identificação de fatores ou indicadores para desnutrição através de uma AN ampla, que inclua revisão da história médica, perfil medicamentoso e nutricional, avaliação bioquímica, condição psicossocial e econômica, prognóstico e desenvolvimento do plano de cuidado médico. Nutrição e exercícios devem estar integrados ao cuidado médico para proteger e restaurar as reservas nutricionais ótimas? ${ }^{7}$.

Segundo Walsek et al. $(1997)^{14}$, é fundamental obter inquérito recordatório de 24 horas de um dia típico ou um diário alimentar detalhado. Deve-se questionar se o apetite e/ou o nível de atividade tem aumentado ou diminuído e se alguma mudança registrada é relativa a um novo ou diferente regime de tratamento ou ao seu estilo de vida.

Conforme observaram Smit \& Tang $(2000)^{18}$, poucos estudos com pacientes soropositivos avaliam a dieta, vários incluem medidas bioquímicas e a maioria utiliza-se de dados antropométricos. Muitas pesquisas sobre consumo alimentar enfocam os tipos de alimentos consumidos, sua quantidade, como são preparados e o padrão de ingestão em um determinado intervalo de tempo. Diversos métodos podem ser utilizados: registro alimentar de sete ou mais dias, recordatórios de 24 horas únicos ou múltiplos, questionários de freqüência de consumo alimentar e história alimentar, traduzindo-se o consumo dos alimentos em nutrientes através de softwares. Estes autores recomendam que os métodos devem ser adaptados cuidadosamente ao grupo abordado ou combinados entre si, pois não há métodos perfeitos.

Luder et al. (1995) ${ }^{19}$ avaliaram o perfil nutricional, clínico e imunológico de pacientes soropositivos, constatando que todas as medidas antropométricas e a contagem de linfócitos CD4 e CD8 foram notavelmente menores nos pacientes com perda de peso. Os registros alimentares indicaram uma ingestão correspondendo a 74\% da recomendação diária. A maioria dos pacientes consumia suplementos de vitaminas e/ou minerais, porém sem efeitos imunológicos positivos significativos, demonstrando que uma alimentação nutritiva e balanceada pode ser mais benéfica para manter ou melhorar o estado fisiológico destes pacientes.

\section{MÉTODOS DE AVALIAÇÃ O DOESTADO NUTRICIONAL}

De acordo com Antunes et al. (1994) ${ }^{20}$, a apreciação do estado nutricional baseia-se atualmente em índices antropométricos e biológicos. Os índices antropométricos abrangem peso total, estatura, composição corporal e suas proporções em gordura e em proteínas, além da determinação do potássio corporal total, normalizado pela altura e idade (importante determinante da MCC). Os índices biológicos compreendem as taxas de certas proteínas plasmáticas (albumina, transferrina e proteína ligante do retinol), assim como a excreção da 
3-metil-histidina urinária. A imunocompetência é um índice que permite efetuar uma aproximação objetiva da desnutrição e correlacioná-la com morbidade e mortalidade nos doentes com AIDS.

Alterações do peso corporal são inespecíficas para determinar qual é o compartimento corporal acometido pela desnutrição ou, ao contrário, em casos de obesidade, podem mascarar uma subnutrição protéica e de micronutrientes. Para Grunfeld \& Kotler (1992)21, a interpretação do peso corporal pode ser dificultada pelos efeitos de diarréia, desidratação, hipoalbuminemia, perda de tecido magro e sobrecarga hídrica. Apesar disso, continua sendo uma informação inestimável em termos de intervenção diagnóstica, terapêutica e nutricional, a qual, juntamente com outros parâmetros, pode identificar deficiências significativas, pois perdas ponderais graves estão associadas com aumento das taxas de morbi-mortalidade dos pacientes.

Entretanto, Shevitz \& Knox $(2001)^{4}$ observam que a relação entre peso atual e peso usual permite um acompanhamento a longo prazo da evolução do peso de um mesmo indivíduo, devendo-se registrar em gráficos de peso seriado.

O exame clínico deve avaliar o estado geral, a força muscular e as medidas das pregas cutâneas, permitindo classificar a desnutrição em leve, moderada ou severa. As dosagens de albumina, cálcio, fósforo, magnésio, vitamina $B_{12}$ folato, vitamina $D$ e zinco completam a relação biológica que deve estar disponível no prontuário do paciente ${ }^{10}$.

Conforme mencionam Walsek et al. $(1997)^{14}$ e Antunes et al. (1994) ${ }^{20}$, as medidas da Prega Cutânea do Tríceps (PCT) e da Circunferência do Braço $(C B)$ freqüentemente evidenciam déficit severo da reserva adiposa em relação ao padrão para o sexo e idade nestes pacientes. A área muscular do braço diminuída indica degradação da proteína muscular, que ocorre juntamente com a depleção de potássio.

Segundo Smit \& Tang (2000) ${ }^{18}$, o método prático mais atual para avaliação da redistribuição de gordura é a medida das circunferências de cintura e quadril, com cálculo da razão cintura-quadril, considerando-se que valores acima de 0,85 para mulheres e acima de 0,95 para homens podem ser indicativos de lipodistrofia e taxas maiores aumentam o risco para diabetes e doença cardiovascular.

Niyongabo et al. (1999a) $)^{3}$ compararam vários métodos de $\mathrm{AN}$ em pacientes adultos infectados pelo HIV, de acordo com a perda de peso corporal, medidas antropométricas, Análise por Bioimpedância (BIA) e Avaliação Nutricional Subjetiva Global (ANSG), a qual inclui parâmetros nutricionais, gastrintestinais e da capacidade funcional. A deterioração do estado nutricional diagnosticada pela ANSG mostrou uma estreita correlação com as classes definidas pelo Centers for Disease Control (CDC) em 1992, forte relação com a perda de peso corporal e boa correlação com todos os indicadores antropométricos, de BIA e bioquímicos (albumina e pré-albumina). A ANSG detectou rapidamente a piora do estado nutricional, sendo benéfica para determinar o uso de TN, enquanto a classificação da desnutrição de acordo com a porcentagem de perda de peso corporal pode detectar a desnutrição mais antecipadamente.

Ao estudarem a prevalência e analisarem as características de desnutrição entre pacientes em Paris, Niyongabo et al. (1997)22 utilizaram um critério comum de classificação a partir da porcentagem de perda de peso em relação ao peso usual (Quadro 2). Foram considerados métodos antropométricos, BIA, albumina e pré-albumina séricas. Dentre os pacientes recrutados, $62,1 \%$ tinham estado nutricional normal; $12,9 \%$, DEP moderada; 16,9\%, DEP intermediária; e $8,1 \%$, DEP severa. Foi observada nos pacientes desnutrição de todas as categorias do CDC, sendo a incidência de $14,9 \%, 27,3 \%$ e $61,8 \%$ para as classes $A, B$ e $C$, respectivamente. Em adição à antropometria, a BIA e a albumina sérica constituíram bons parâmetros para diagnosticar, tratar e acompanhar a DEP. 
Quadro 2. Classificação da desnutrição de acordo com a perda de peso.

\begin{tabular}{ll}
\hline Perda de peso & Estado nutricional \\
\hline Menor ou igual a $5 \%$ do peso usual & Sem desnutrição \\
Entre $5 \%$ e $10 \%$ do peso usual & Desnutrição moderada \\
Entre $10 \%$ e $20 \%$ do peso usual & Desnutrição intermediária \\
Maior que $20 \%$ do peso usual & Desnutrição severa \\
\hline
\end{tabular}

Considerando os mesmos critérios clínicos e bioquímicos, Niyongabo et al. (1999) ${ }^{23}$ observaram a prevalência, as características e as causas de DEP entre pacientes na África. Dos indivíduos estudados, 45,1\% eram soropositivos, e destes, 60,8\% eram casos de AIDS. Dentre todos os pacientes, o estado nutricional foi normal em $52,7 \%$, havendo DEP moderada em $20,8 \%$ e DEP severa em $26,5 \%$. A soroprevalência do HIV e a porcentagem dos casos de AIDS aumentaram de acordo com a diminuição do nível de nutrição. Entre os soropositivos a DEP esteve associada à tuberculose e disfagia, e nos soronegativos relacionou-se com a baixa disponibilidade de alimentos, mostrando o impacto da pobreza e das dificuldades políticas no estado nutricional da comunidade, muito pior quando comparado aos países desenvolvidos.

Hoh et al. (1998) ${ }^{24}$ estudaram pacientes com perda de peso associada ao HIV, considerando uso de suplementos enterais nas ingestões protéica e energética, composição corporal, gasto energético e histologia gastrintestinal. Não foram notadas diferenças significativas quanto a todos os parâmetros entre os dois grupos suplementados, porém as ingestões protéica e energética foram superiores no grupo dos não suplementados. Houve significativa diferença no ganho de MCM durante a suplementação entre os indivíduos com menor lipogênese "de novo" e perda de MCM naqueles com maior lipogênese "de novo", refletindo a ação das citoquinas nos pacientes com síndrome consumptiva.

Avaliando pacientes assintomáticos para determinar o efeito de alterações metabólicas e nutricionais na progressão da doença em estágios precoces da infecção pelo HIV, Sharpstone et al.
(1999) ${ }^{25}$ observaram que houve uma resposta caquética em todos os compartimentos corporais e aumento significativo da oxidação de gordura e da Taxa Metabólica Basal (TMB), associados a um diagnóstico definidor de AIDS. O risco para progressão da doença foi similar em pacientes com diferentes pesos, todavia, foi maior nos pacientes com perda de peso ou queda do Índice de Massa Corporal (IMC), em relação àqueles com uma composição corporal mais estável, independentemente da contagem de CD4.

A discriminação precoce de alterações da composição corporal deve usar métodos reprodutíveis, não invasivos e de custo acessível. Em sujeitos infectados pelo HIV, a depleção da MCC é proporcionalmente mais importante que as reduções em peso e gordura corporais. A BIA possibilita detectar modificações precoces da composição corporal no curso da infecção; porém, é muito dependente da variação hídrica. A avaliação por DEXA permite direcionar novas estratégias terapêuticas para tratar a infecção viral, aumentar a MCM, estimular o apetite e até inibir a ação das citoquinas 26,27 .

A MCC determinada pelo conteúdo total de potássio corporal não é afetada por alterações no balanço fluido, como ocorre com a determinação da MCM através de técnicas antropométricas ou de BIA. Enquanto a dosagem do potássio é sofisticada e cara, a BIA é uma técnica facilmente aplicada na prática clínica, correlacionando-se bem com métodos tradicionais, como potássio corporal total, densitometria, antropometria e métodos de diluição de isótopos, tanto em indivíduos saudáveis, quanto em pacientes portadores de diferentes doenças e com estados nutricionais diversos. Na prática clínica, a calorimetria indireta não está disponível, enquanto a determinação das pregas cutâneas é simples ${ }^{12}$.

Há vários métodos de avaliação da MCM disponíveis na prática clínica, para pacientes com HIV/AIDS, mencionados por Shevitz \& Knox $(2001)^{4}$ e Corcoran \& Grinspoon (1999) ${ }^{28}$, apresentando vantagens e desvantagens: 
- Medida das pregas cutâneas - facilmente desenvolvida com equipamento portátil, com possibilidade de erro e variabilidade na medição entre os observadores, exigindo treinamento cuidadoso e padronização;

- Análise por bioimpedância - facilmente desenvolvido com equipamento portátil e de baixo custo, é usado para calcular a MCC, porém os resultados podem ser afetados pela hidratação, cafeína, álcool, atividade física anterior à medição e ainda não estão validados com o uso de terapia antiretroviral altamente ativa (HAART);

- Dual Energy X-ray Absorptiometry (DEXA) - método não invasivo, com quantidade mínima de radiação, considerado como a melhor ferramenta de avaliação da composição corporal, mas é cara, os resultados podem ser afetados por hidratação e calcificação, e as medidas regionais não estão padronizadas;

- Pletismografia de corpo inteiro - método de densitometria no qual o volume corporal é medido em uma câmara fechada, entretanto é complexo e de alto custo;

- Tomografia computadorizada e imagem por ressonância magnética do abdômen, coxa ou corpo total têm sido usadas, no entanto cada qual apresenta riscos como radiação e claustrofobia e só podem ser analisadas por especialistas treinados. Requerem equipamentos caros e os valores normais não estão estabelecidos para a adiposidade visceral.

Entre os exames bioquímicos relativos à nutrição, Walsek et al. (1997) ${ }^{14}$ citam que normalmente são incluídos hemograma completo, dosagem de eletrólitos, prova da função hepática (TGO e TGP), prova da função renal através da determinação da uréia e creatinina séricas, dosagem das proteínas séricas (albumina, transferrina e proteínas totais) e avaliação da integridade gastrintestinal, através de pesquisa de sangue oculto e parasitológico de fezes. Os parâmetros a serem monitorados e sua freqüência devem ser considerados em relação ao estágio da doença, estado clínico geral e regime medicamentoso.
Perda de peso e baixos níveis séricos de albumina são indicativos de aumento no risco de morbidade e mortalidade nos pacientes com AIDS hospitalizados e desempenham papel crítico na evolução da infecção pelo HIV. Conforme relataram Antunes et al. (1994) ${ }^{20}$, estudos têm demonstrado que a freqüência de complicações aumenta rapidamente quando os níveis de albumina sérica estão abaixo de 3,0 g/dL. Existe progressiva depleção da MCC em pacientes perto da morte, avaliada pelo conteúdo de potássio corporal total, com nível aproximado de $54 \%$ do normal. A perda progressiva de peso corporal relaciona-se com a morte, chegando a até $66 \%$ do peso corporal usual.

Del-Olmo et al. (1997) ${ }^{16}$ avaliaram pacientes com HIV/AIDS, na internação e na alta, em relação a parâmetros antropométricos e bioquímicos. Foi registrada uma perda de peso prévia em $85 \%$ dos pacientes, sendo severa em $39,6 \%$, moderada em $15,1 \%$ e leve em $30,2 \%$. De acordo com a AN inicial 98,3\% dos pacientes estavam desnutridos e segundo a avaliação final $90,5 \%$ continuaram desnutridos. Não houve diferenças significativas em nenhum dos parâmetros avaliados, exceto em relação à pré-albumina e transferrina, as quais aumentaram devido ao controle da infecção. Os pacientes não receberam TN durante a internação, comprovando que a recuperação espontânea é incompleta e parcial.

A deterioração do estado nutricional em pacientes infectados pelo HIV é complicada pelas mudanças associadas à doença na estrutura e função gastrintestinal, bem como pelos efeitos adversos de drogas antiretrovirais. As proteínas séricas totais ${ }^{9}$ podem estar aumentadas como resultado da hiperimunoglobulinemia por ativação das células B. A albumina também pode ser influenciada por fatores não-nutricionais, como fluidos intravenosos, insuficiência renal ou hepática ou resposta de fase aguda à injúria. Um baixo nível de colesterol (abaixo de $100 \mathrm{mg} / \mathrm{dL}$ ) é indicativo de DEP e síndrome consumptiva ou wasting syndrome (WS) ${ }^{13}$. 
No estudo desenvolvido por Silveira et al. $(1999)^{29}$ no Brasil, foi avaliado o estado nutricional global e os níveis de vitamina $A$. Os resultados mostraram déficit ponderal de $89,9 \%$ nos pacientes com AIDS, contra $105,4 \%$ de peso ideal no grupo HIV e $123,5 \%$ no grupo-controle (indivíduos saudáveis). Dos pacientes com AIDS, $78,1 \%$ apresentavam IMC abaixo dos valores normais e a PCT mostrou-se bastante alterada, correspondendo a $55,1 \%$ do ideal, enquanto foi de $89,9 \%$ no grupo HIV e de $131,8 \%$ no grupo-controle. O Índice de Gordura do Braço (IGB) também foi significativamente reduzido nos pacientes com AIDS em relação aos outros grupos. A circunferência muscular do braço esteve relativamente bem preservada, sem diferença estatística entre os mesmos. Esses resultados, em adição aos observados pela dosagem de albumina sérica e o índice creatinina altura, permitiram classificar o padrão de desnutrição predominante na AIDS como tipo marasmo. Quanto aos níveis de vitamina $A$, foram verificados déficit de $25,0 \%$ nos pacientes com AIDS e de 17,3\% nos soropositivos e baixa reserva corporal em $28,0 \%$ dos pacientes, independentemente de apresentarem ou não imunodeficiência significativa.

\section{RECOMENDAÇÕ ES GERAIS}

Recomenda-se que todo paciente infectado pelo HIV seja encaminhado ao profissional nutricionista logo após o primeiro diagnóstico para avaliação do seu perfil nutricional, determinando e priorizando intervenções dietoterápicas apropriadas através de metas individualizadas, para tratar deficiências nutricionais, manter ou restaurar a MCM e melhorar a qualidade de vida.

É fundamental o tratamento das doenças agudas para que a TN consiga reverter ou amenizar o processo de consumo. A própria terapia medicamentosa pode induzir a transtornos gastrintestinais, anorexia e conseqüente perda de peso. Portanto, seus horários devem ser temporizados para o máximo proveito tanto dos medicamentos como dos alimentos, otimizando a qualidade de vida do paciente. Deve-se considerar também a relação custo-benefício do tratamento.

A desnutrição e seus distúrbios devem ser prevenidos e/ou tratados através de metas eficientes apoiadas em três bases: avaliação, orientação e intervenção nutricional. A complexidade da avaliação, o tipo da orientação e o grau de intervenção devem variar com o estágio de doença do indivíduo.

Muitos estudos têm sido desenvolvidos, envolvendo indivíduos infectados em diferentes estágios e utilizando diferentes tipos de TN, com critérios precisos de inclusão e exclusão, grupos-controle e métodos de avaliação específicos e validados. A uniformidade de condutas e a divulgação dos resultados propiciam um melhor direcionamento de esforços para restaurar a composição corporal e prováveis carências nutricionais.

\section{REFER Ê NCIAS}

1. Ministério da Saúde. AIDS. Bol Epidemiol-AIDS 2001; 14(2):3-14.

2. Kotler DP. Nutritional effects and support in the patient with acquired immunodeficiency syndrome. J Nutr 1992; 122:723-27.

3. Niyongabo T, Melchior JC, Henzel D, Bouchaud O, Larouzé B. Comparison of methods for assessing nutritional status in HIV-infected adults. Nutrition 1999; 15(10):740-43.

4. Shevitz AH, Knox TA. Nutrition in the era of highly active antiretroviral therapy. Clin Infect Dis 2001; 32(12):1769-75.

5. Singer $P$, Katz DP, Dillon $L$, Kirvelä $O$, Lazarus $T$, Askanazi J. Nutritional aspects of the acquired immunodeficiency syndrome. Am J Gastroenterol 1992; 87(3 Suppl):265-73.

6. Gramlich LM, Mascioli EA. Nutrition and HIV infection. J Nutr Biochem 1995; 6(1):2-11.

7. American Dietetic Association. Position of the American Dietetic Association and the Canadian 
Dietetic Association: nutrition intervention in the care of persons with human immunodeficiency virus infection. J Am Diet Assoc 1994; 94(9 Suppl):1042-5.

8. Carr T. Nutrition in HIV and AIDS. Nursing Times 1997; 93(36 Suppl):S1-S6.

9. McCorkindale C, Dybevik K, Coulston AM, Sucher KP. Nutritional status of HIV-infected patients during early disease stages. J Am Diet Assoc 1990; 90(9):1236-41.

10. Babameto G, Kotler DP. Malnutrition in HIV infection. Gastroenterol Clin N Am 1997; 26(2):393-415

11. Melchior JC, Raguin G, Boulier A, Bouvet E, Rigaud $D$, Matheron $S$, et al. Resting energy expenditure in human immunodeficiency virus-infected patients: comparison between patients with or without secondary infections. Am J Clin Nutr 1993; 57:614-9.

12. Ott $M$, Lembecke $B$, Fischer $H$, Jäger $R$, Polat $R$, Geier $H$, et al. Early changes of body composition in human immunodeficiency virus-infected patients: tetrapolar body impedance analysis indicates significant malnutrition. Am J Clin Nutr 1993; 57:15-9.

13. Davidhizar R, Dunn C. Nutrition and the client with AIDS. J Prac Nurs 1998; 48(1):16-28.

14. Walsek C, Zafonte M, Bowers JM. Nutritional issues and HIVIAIDS: assessment and treatment strategies. J Assoc Nurs AIDS Care 1997; 8(6): 71-80.

15. Carbonnel F, Cosnes J. Dénutrition protéino-énergétique et VIH. Impact Médecin Guide SIDA 1997: Les dossiers du praticien. Paris; 1997. p.121-5.

16. Del-Olmo D, Alcázar V, Barba R, Fernandez C, Koning MA, Vásquez C. Estado nutricional de pacientes con infección por VIH. Evolución espontánea durante un ingreso hospitalario. Nutr Hosp 1997; 12(4):206-9.

17. Macallan DC, Noble C, Baldwin C, Jebb SA, Prentice AM, Coward $A$, et al. Energy expenditure and wasting in human immunodeficiency virus infection. N Eng J Med 1995; 333(2 Suppl):83-8.

18. Smit E, Tang A. Nutritional assessment in intravenous drug users with HIVIAIDS. J Acquir Immune Defic Syndromes 2000; 25(1 Suppl): S62-9.

19. Luder E, Godfrey E, Godbold J, Simpson DM. Assessment of nutritional, clinical, and immunologic status of HIV-infected, inner-city patients with multiple risk factors. J Am Diet Assoc 1995; 95(6):655-60.

20. Antunes MC, Silva MA, Lima MBC. Determinantes do estado nutricional em pacientes com Aids. J Bras Med 1994; 67(4):209-22.

21. Grunfeld C, Kotler DP. Wasting in the acquired immunodeficiency syndrome. Sem Liver Dis 1992; 12(2 Suppl):175-87.

22. Niyongabo T, Henzel $D$, Ndayishimyie JM, Melchior JC, Ndayiragije A, Ndihokubwayo JB, et al. Nutritional status of adults inpatients in Bujumbura, Burundi (impact of HIV infection). Eur J Clin Nutr 1999; 53(7):579-82.

23. Niyongabo T, Bouchaud O, Henzel D, Melchior JC, Samb B, Dazza MC, et al. Nutritional status of HIVseropositive subjects in an AIDS clinic in Paris. Eur J Clin Nutr 1997; 51(9):637-40.

24. Hoh R, Pelfini A, Neese RA, Chan M, Cello JP, Cope $F O$, et al.De novo lipogenesis predicts short-term body-composition response by bioelectrical impedance analysis to oral nutritional supplements in HIV-associated wasting. Am J Clin Nutr 1998; 68:154-63.

25. Sharpstone D, Murray C, Ross H, Phelan M, Crane $R$, Lepri $A C$, et al. The influence of nutritional and metabolic status on progression from asymptomatic HIV infection to AIDS-defining diagnosis. AIDS 1999; 13(10):1221-6.

26. Bell SJ, Bistrian BR, Connolly CA, Forse RA. Body composition changes in patients with human immunodeficiency virus infection. Nutrition 1997; 13(7-8):629-32. 
27. Gilquin B, Marchandise X. Composition corporelle ao cours de l'infection à $\mathrm{VIH}$ : physiopathologie, méthodes de mesure et revue des résultats. Rev Méd Int 1997; 18(10):786-94.

28. Corcoran C, Grinspoon S. Treatments for wasting in patients with the acquired immunodeficiency syndrome. N Eng J Med 1999; 340(22):1740-50.
29. Silveira SA, Figueiredo JFC, Jordão-Júnior AA, Unamumo MRD, Rodrigues MLV, Vannucchi $H$. Subnutrição e hipovitaminose A em pacientes com AIDS. Rev Soc Bras Med Trop 1999; 32(2):119-24.

Recebido para publicação em 5 de dezembro de 2001 e aceito em 27 de fevereiro de 2003. 